\title{
DESIGN, FABRICATION, AND TEST OF WFIRST/AFTA GRISM ASSEMBLY
}

Qian Gong, David Content, Jeffrey Kruk, Bert Pasquale, Thomas Wallace, and Walter Smith NASA Goddard Space Flight Center 


\section{WFIRST-AFTA: A Unique Probe of Cosmic Structure Formation History}

Using Observations from the High Latitude Survey and GO Programs

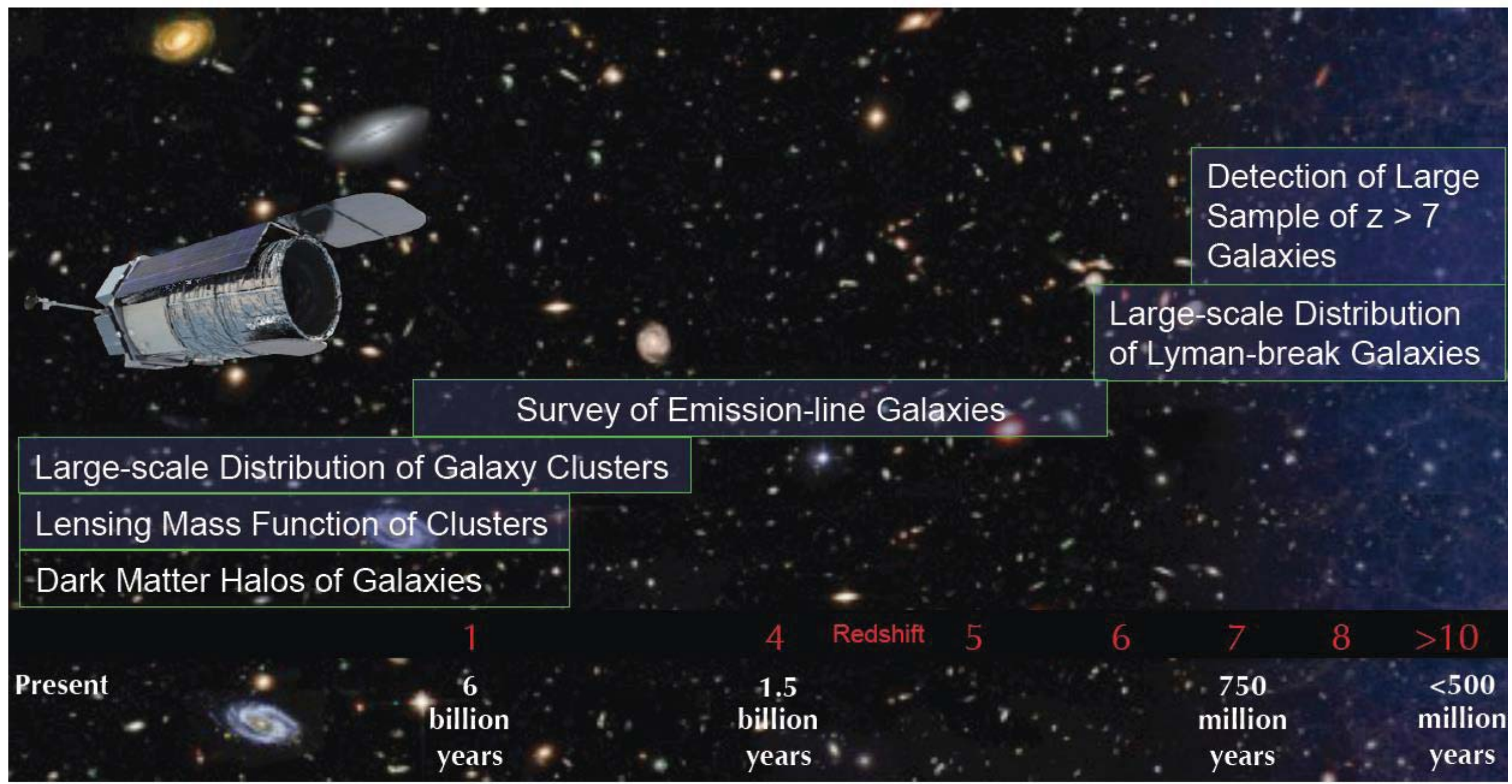




\section{$\frac{\text { WITUR - T Wide Field Infrared Survey Telescope - AFA }}{\text { WFIST/AFTA \& GRISM ASSEMBLY }}$}

- Wide-Field Infra-Red Survey Telescope (WFIRST) is designed to perform wide-field imaging and slitless spectroscopic survey of the sky.

- A compound grism assembly is selected as its slitless dispersing element.

- The challenge of this grism is the wider Field Of View (FOV), larger dispersion, and smaller f/\# .

- The challenge is overcome by the innovative design of using two diffractive surfaces. 


\section{WDIDR - T Wide Field Infrared Survey Telescope = AFTA Nash}

\section{GRISM Comparison: WFIRST versus HST}

- Grisms have been used in a number of Hubble Space Telescope (HST) instruments: Wide Field Camera 3 (WFC3), Near Infrared Camera and Multi-Object Spectrometer (NICMOS), Advanced Camera for Surveys (ACS), etc.

- There are 3 differences that makes WFIRST grism much more challenge than HST's:

\begin{tabular}{|l|l|l|}
\hline & WFIRST & HSTWFC3 \\
\hline FOV & 0.28 degree $^{2}$ & 0.0012 degree $^{2}$ \\
\hline Spectral resolution & $R=700$ & $R=130$ \\
\hline f/\# & 8 & $\mathrm{II}$ \\
\hline
\end{tabular}




\section{WVIDR - TR Wide Field Infrared Survey Telescope - AFTA NAg}

\section{WFIRST GRISM SPECIFICATION}

Wavelength range $(\mu \mathrm{m}) \quad 1.35-1.95$

FOV $\left(^{\circ}\right)$

$0.788 \times 0.516$

Beam diameter at grism 120

( $\mathrm{mm}$ )

Beam f/-ratio at grism $\quad \sim f / 8$

( $\mathrm{mm}$ )

Wavefront error

Diffraction limited at $1.65 \mu \mathrm{m}$

Spectral resolving power $645-900(461 \times \lambda)$

(per 2 pixels)

Compactness

$70 \mathrm{~mm}$ total thickness for a fixed diameter $\sim 120 \mathrm{~mm}$ 


\section{DESIGN CONSIDERATION}

- The main challenge of the grism design is how to correct the grating introduced aberration in noncollimated space? Besides, it needs to be compact enough to fit into a filter wheel slot. It also needs to be designed parforcal to other filters.

- The aberration is huge at large incident angles $\left( \pm 12.5^{\circ}\right)$ and the amount of aberration is proportional to wavelength.

- The wavelength dependent aberration is very difficult to correct using non-diffractive lenses, even with many freeform surfaces.

- The key is to find a right compensator: another diffractive surface "diffractive lens" 


\section{Wide Field Infrared Survey Telescope = AFTA}

\section{GRISM OPTICAL DESIGN}
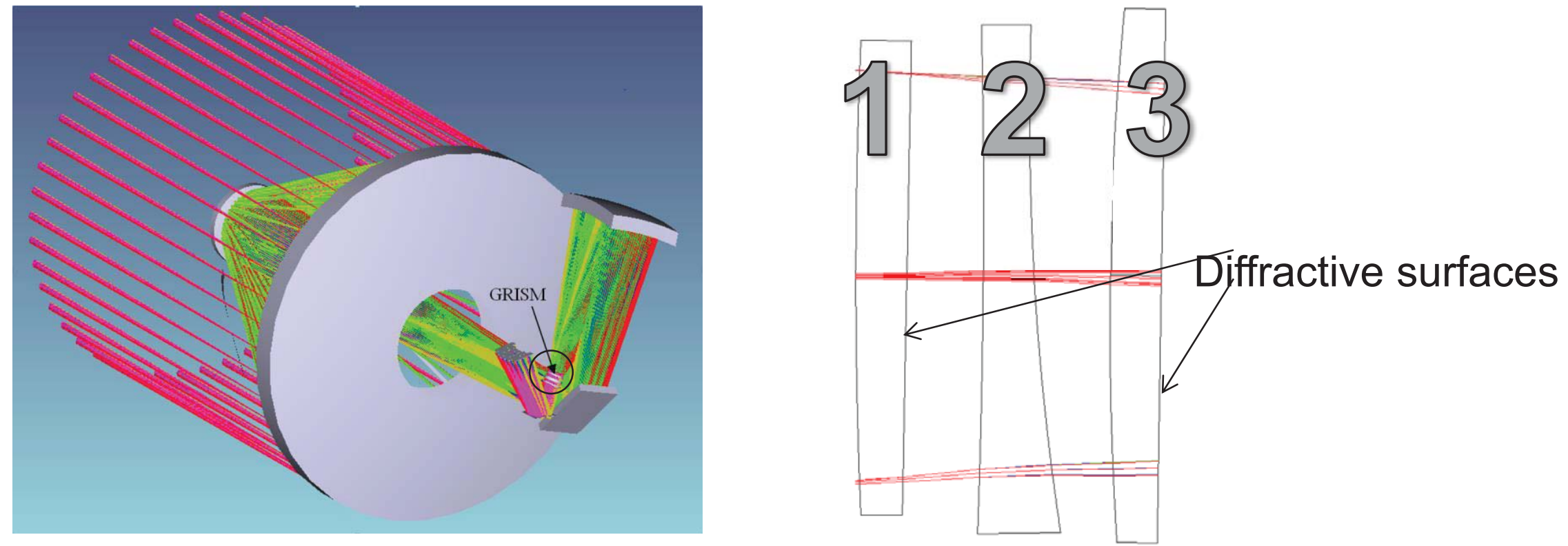

Element 1:

Substrate: fused silica

Function: bandpass filter and aberration corrector

$\mathrm{S} 1$ is sphere, and S2 is diffractive lens on flat
Element 2:

Substrate: fused silica

Function: prism to make grism zero deviation

Both S1 and S2 are spheres

\section{Element 3:}

Substrate: fused silica

Function: grating to provide required dispersion

$\mathrm{S} 1$ is sphere, and S2 is grating on flat 


\section{WITIR - T Wide Field Infrared Survey Telescope - AFTA NAgh}

\section{GRISM IN WFIRST ELEMENT WHEEL}

- WFIRST/AFTA has a number of subassemblies, the element wheel is one of the major assemblies.

- Grism assembly is one element in the element wheel.

- Grism has to be compact enough in thickness to avoid conflict with the beams and other subassemblies.

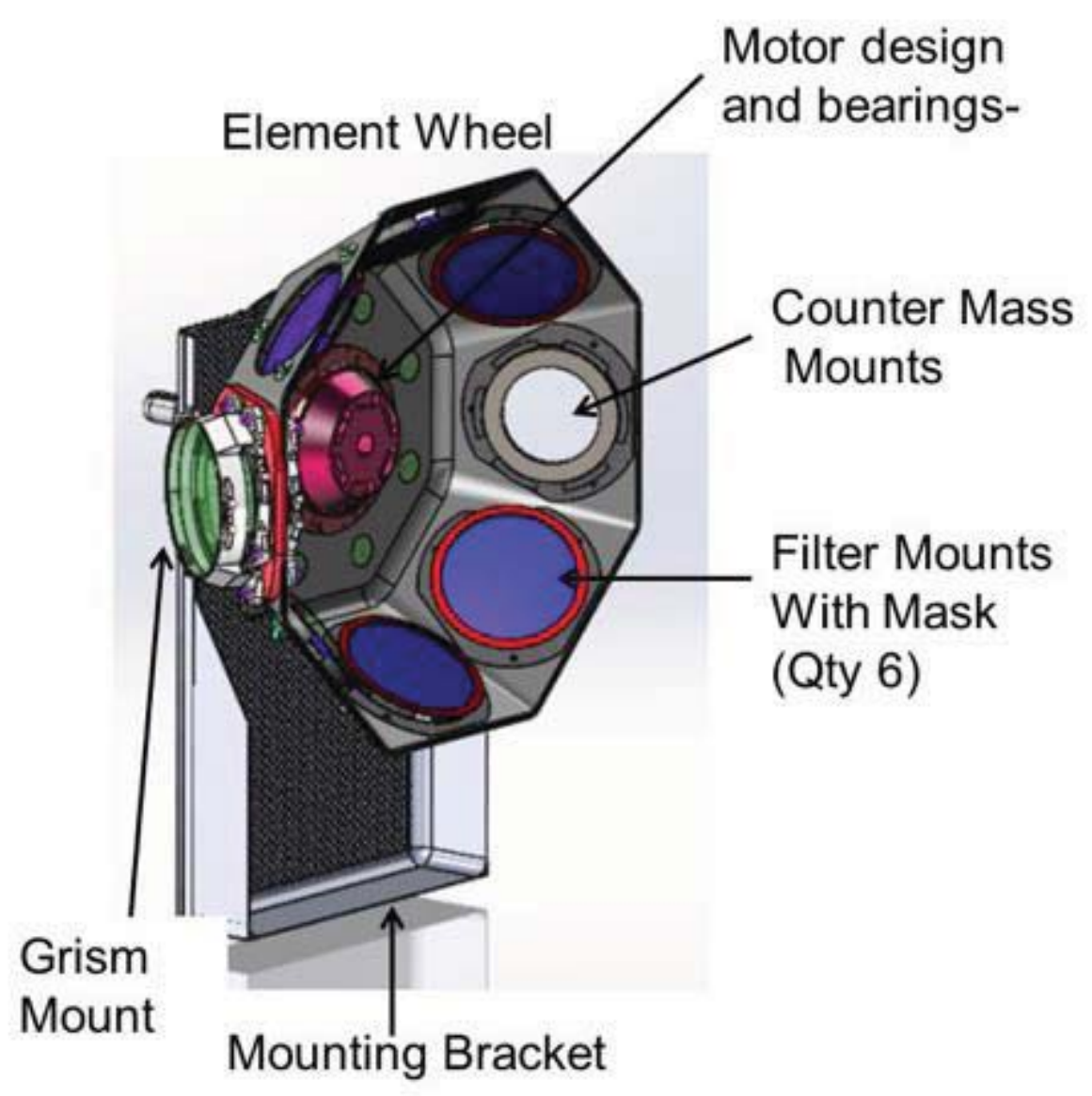




\section{GRISM MATERIAL SELECTION}

- Fused silica is selected for all grism elements. Its property is well know and well characterized.

- The diffractive patterns will be ion etched into the lens substrate. The fused silica is one of the best choices for reactive ion etching.

- Because of the more reproducible and small temperature derivative of refractive index of the fused silica, the fabrication and alignment tolerance are greatly relaxed.

- Fused silica has been well characterized at WFIRST operation temperature $170^{\circ} \mathrm{K}$. 


\section{W】}

\section{DIFFRACTION LIMITED PERFORMANCE}

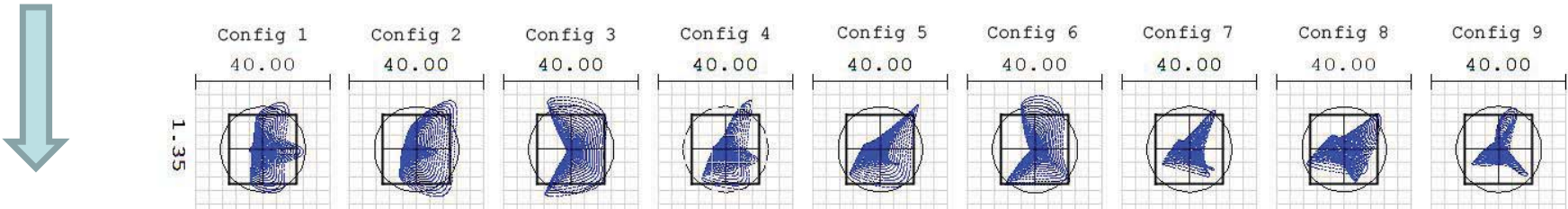

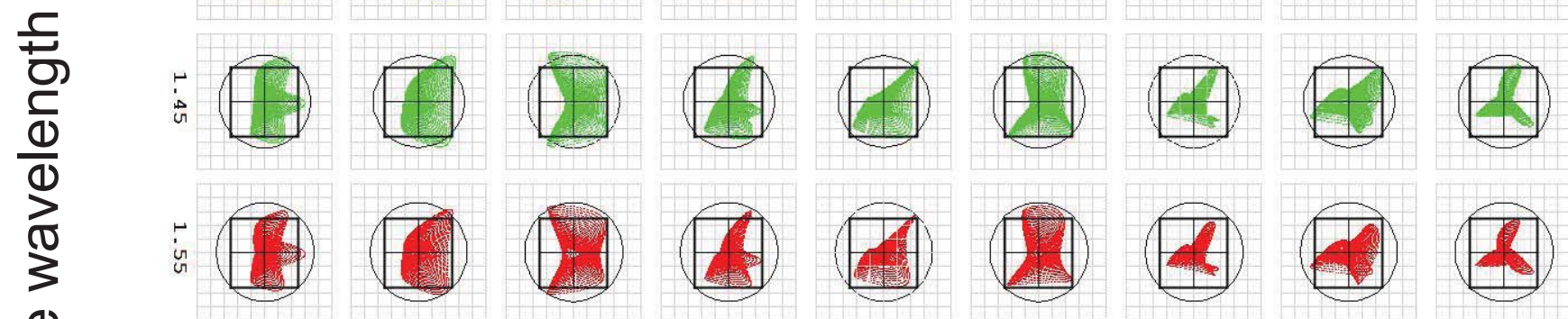

\begin{tabular}{l}
1 \\
\multirow{2}{0}{} \\
0 \\
0 \\
0 \\
$\frac{0}{4}$
\end{tabular}

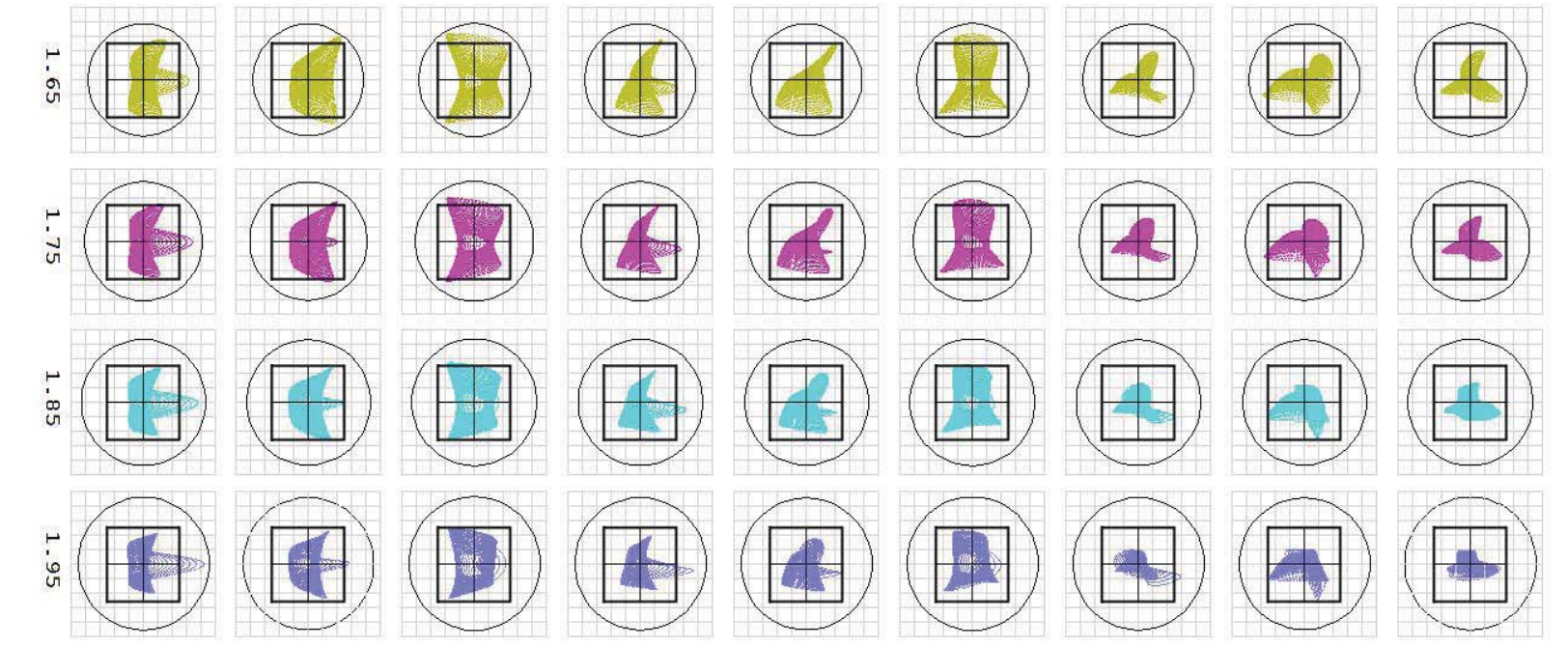




\section{SPECTRAL RESOLVING POWER MEETS SPECS.}

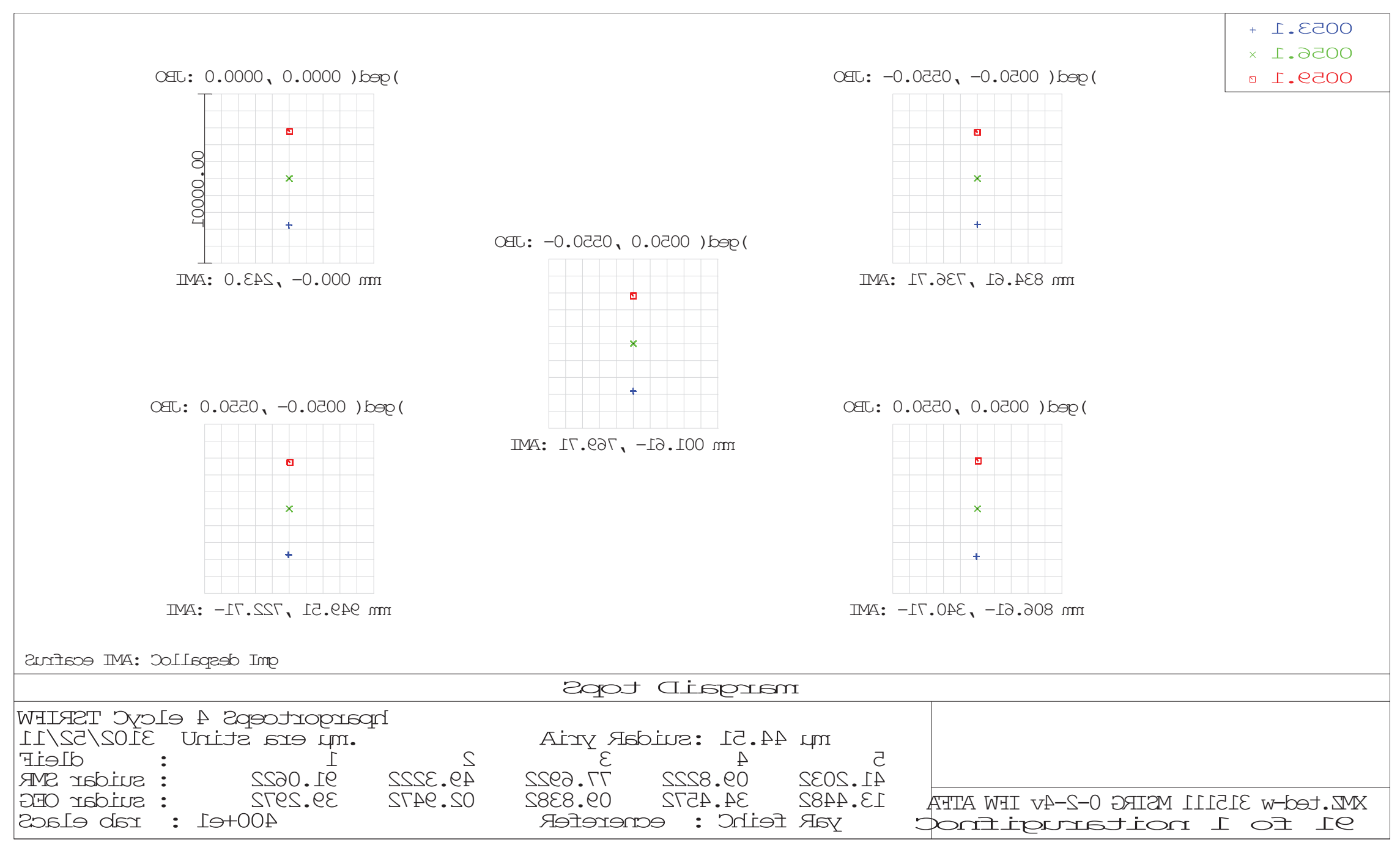




\section{REMOVE UNWANTED GRATING ORDERS TO BACKGROUND}

\section{Spot diagram for different diffractive lens orders}

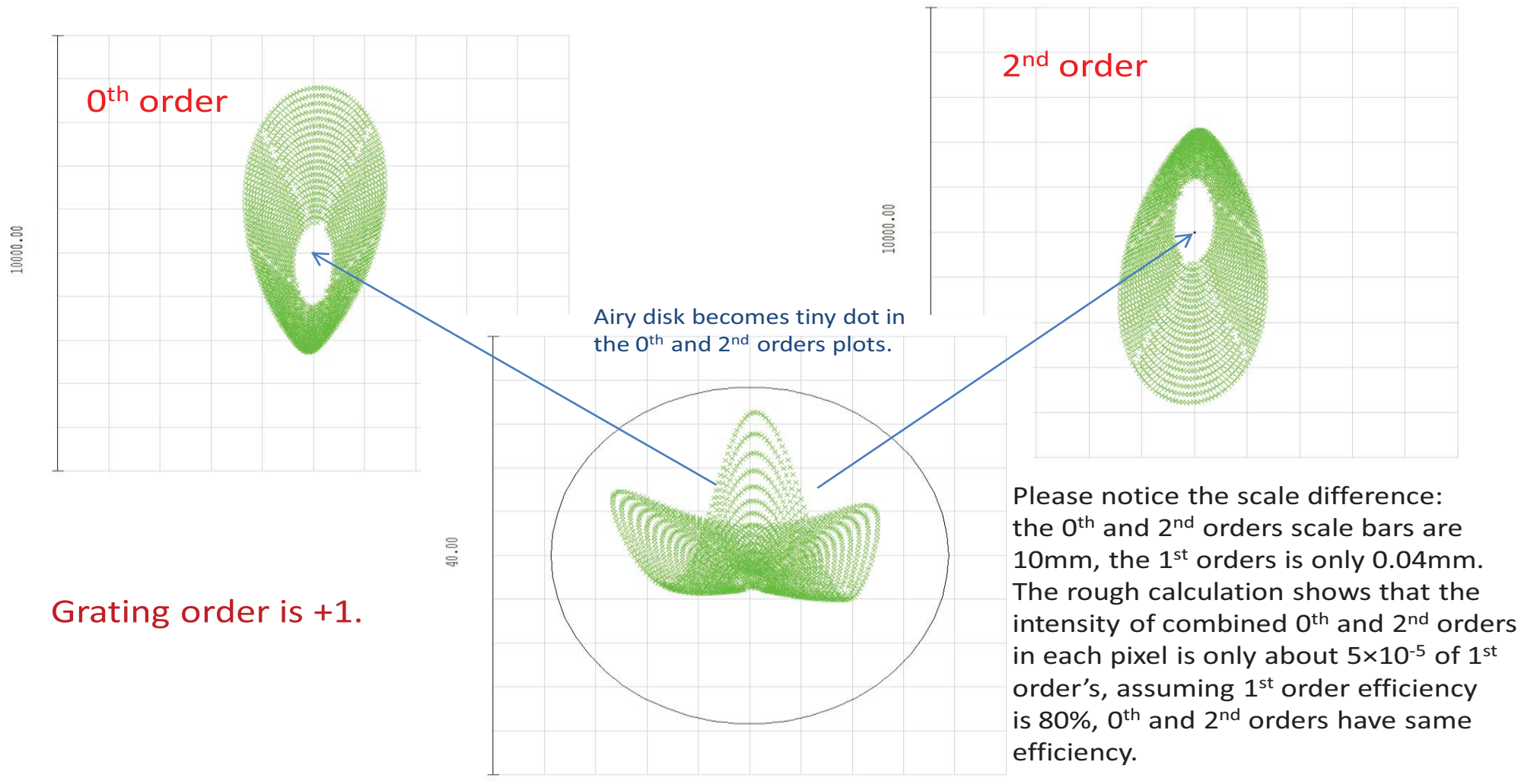

$1^{\text {st }}$ order: fabricated to include $>90 \%$ energy 


\section{PATTERNS OF TWO DIFFRACTIVE SURFACES}

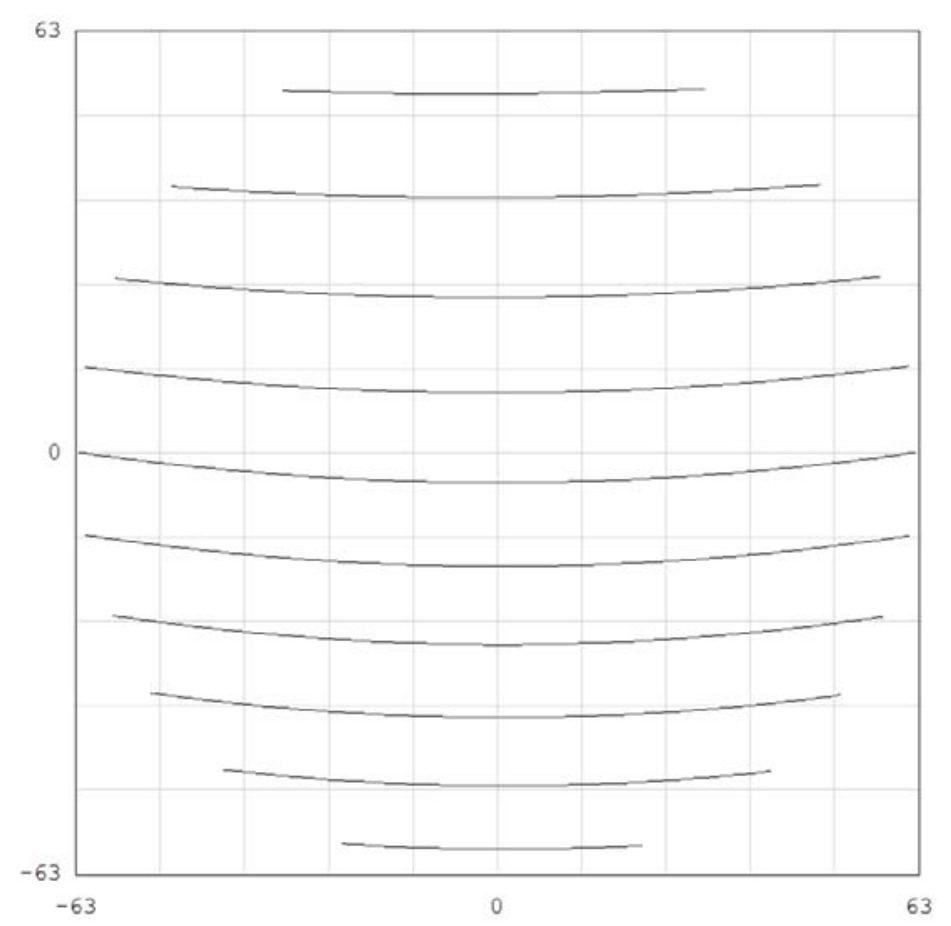

Grating contour. There are 165 lines between the two plotted lines.

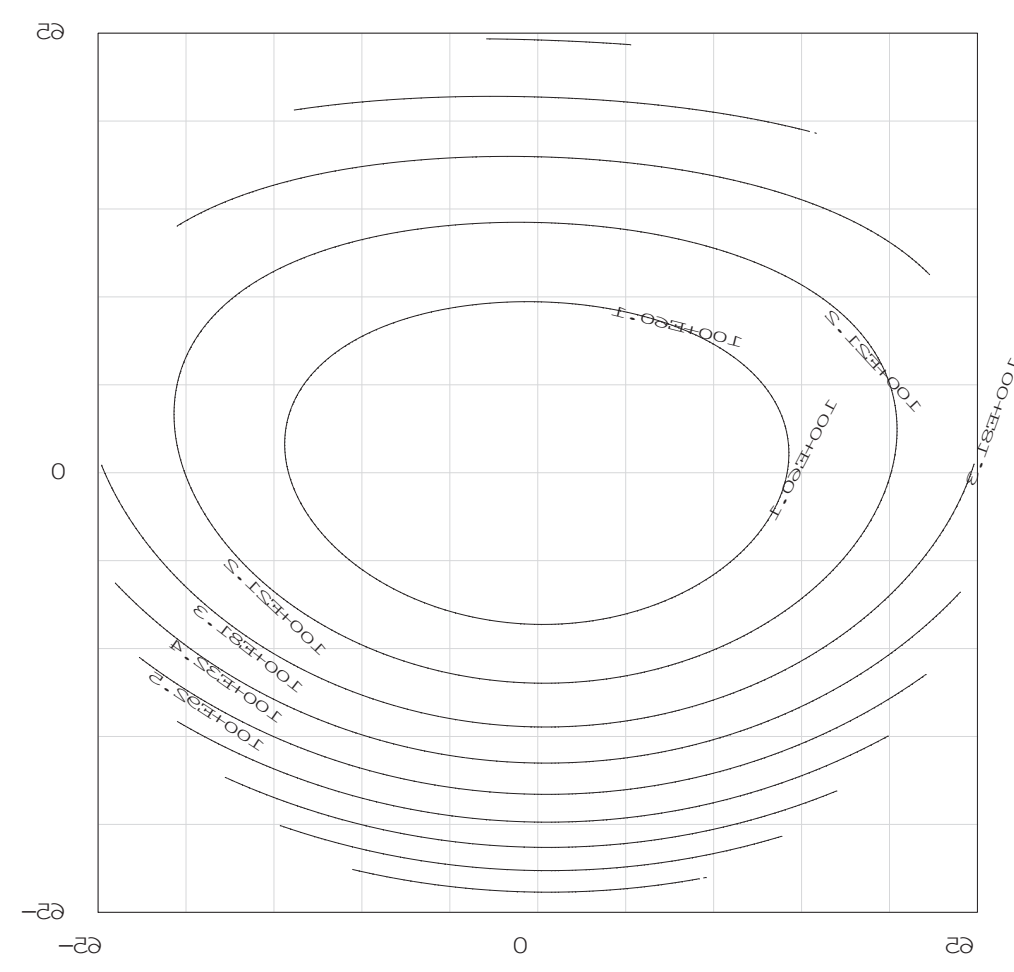

Diffractive lens contour. There are $\sim 10$ lines between the two plotted lines. 


\section{WVIDR - T Wide Field Infrared Survey Telescope - AFTA Nash}

\section{ASSEMBLY'S MECHANICAL DESIGN}
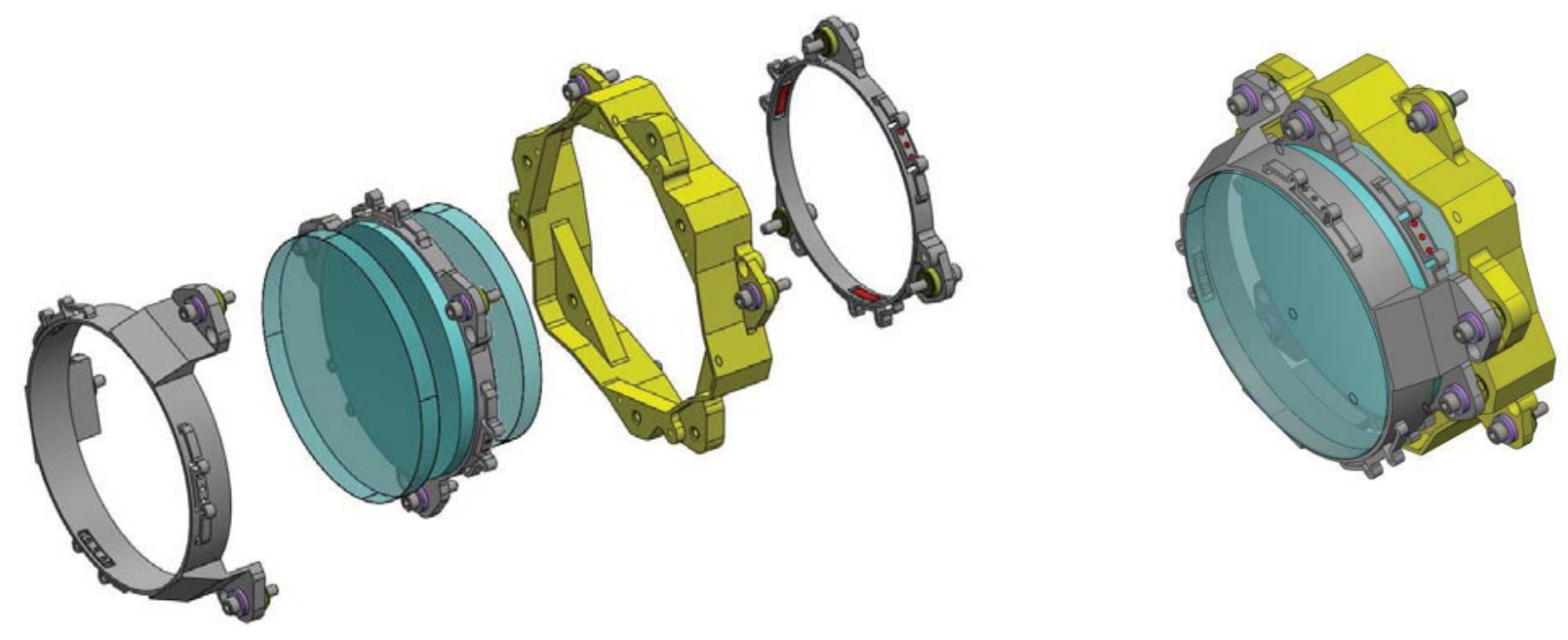

- Titanium is selected as element mount material to minimize the wavefront distortion at $170^{\circ} \mathrm{K}$.

- A ring structure tested in similar environment for another project is used to further control CTE mismatch introduced wavefront degradation.

- 5 degrees of freedom are designed into two of the three elements to provide desired adjustment. 


\section{WIIIR - W Wide Field Infrared Survey Telescope = AFTA NAsA}

\section{DIFFRACTION EFFICIENCY OF SAMPLES}

- Four diffractive samples have been made in one fused silica wafer.

- The samples were designed for HeNe wavelength at $632.8 \mathrm{~nm}$ in order to simplify the measurement.

- There are two diffractive lenses and two grating patterns on the wafer.

- The measured results show the diffraction efficiency is over $90 \%$.

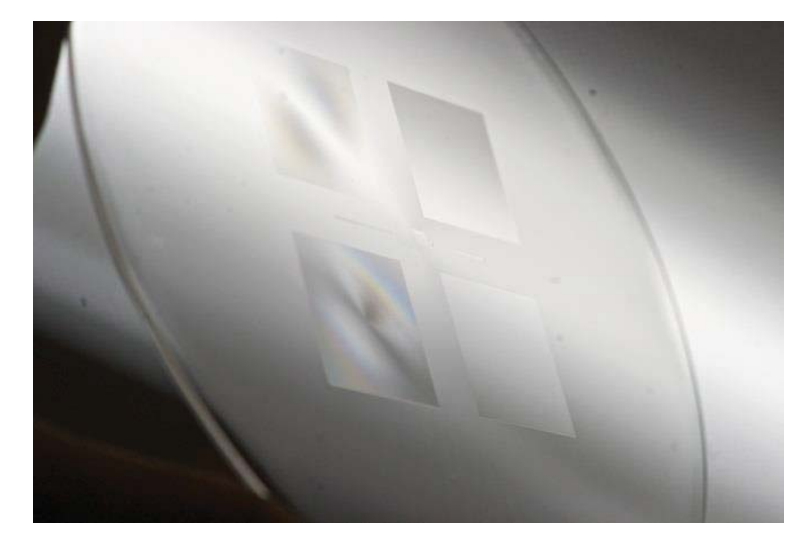


GRISM TEST CONFIGURATION

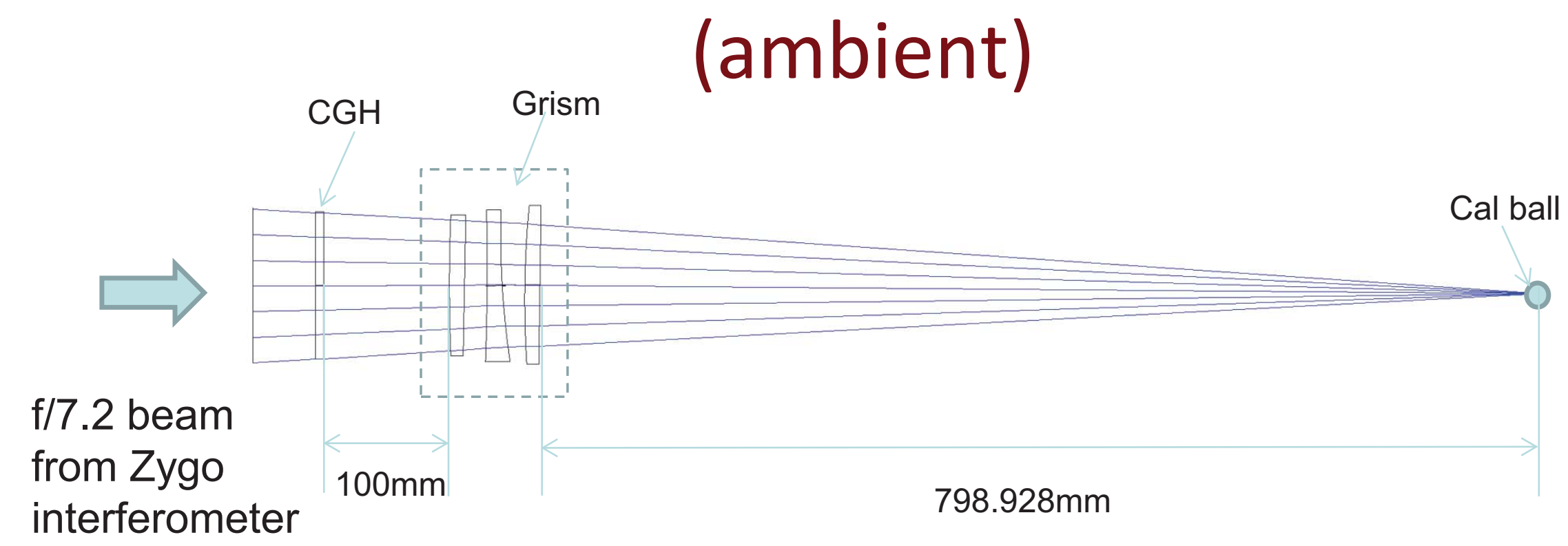

- Ambient test will be performed using interferometer.

- Even though the grism is designed for NIR wavelength, it can be tested in visible at $632.8 \mathrm{~nm}$ with the help of a specially designed Computer Generated Interferogram (CGH). 


\section{WVIDR - TR Wide Field Infrared Survey Telescope - AFTA NAgh}

\section{GRISM TEST CONFIGURATION (cryogenic)}

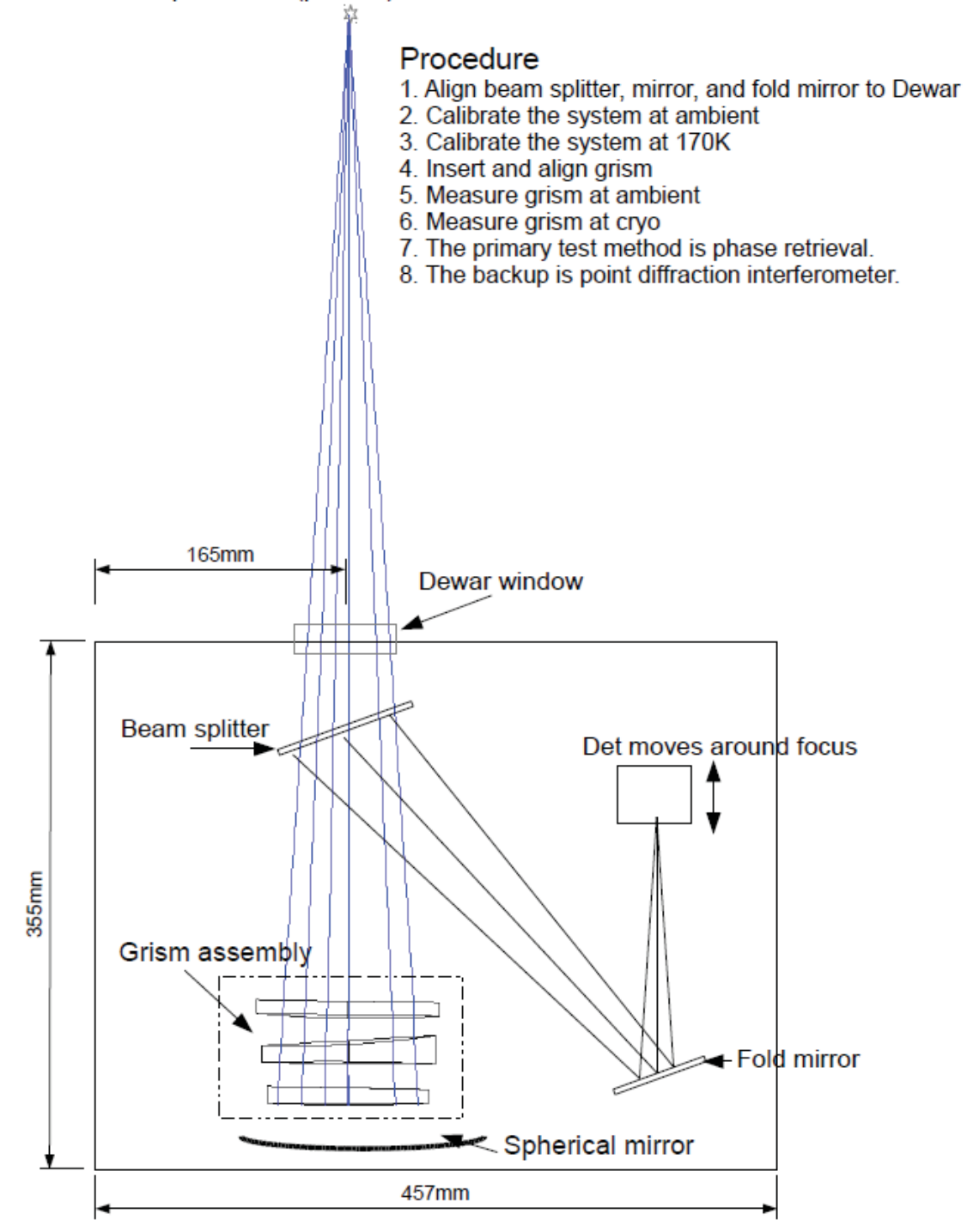

- Phase retrieval will be used for final assembly test at cryogenic temperature at $170^{\circ} \mathrm{K}$.

- A few IR wavelengths between $1.35 \mu \mathrm{m}$ and $1.95 \mu \mathrm{m}$ will be used during the test. 


\section{CONCLUSION}

- The analysis shows that the grism with two diffractive surfaces solves the previous problem: grism is limited to small FOV, small spectral dispersion, and in non-collimated space.

- All three lens substrates have been made. We are working with vendors (RPC Photonics and JenOptik) to etch the diffraction patterns into the two flat surfaces. 